\title{
MATHEMATIKA
}

A JOURNAL OF PURE AND APPLIED MATHEMATICS

Vol. 45, PART 2

December, 1998

No. 90

\section{NONLINEAR EVOLUTION OF RAYLEIGH WAVES IN AN INITIAL VALUE CONTEXT: NON-SYMMETRIC INPUT AND CROSS-FLOW}

\author{
T. ALLEN, S. N. BROWN AND F. T. SMITH
}

Abstract. In recent papers the present authors considered the effects of small cross-flow on the evolution of two unequal oblique waves. In these studies the relative size of the crossflow meant that a diffusion (or buffer) layer was required around the critical layer to smooth out the algebraic growth in the mean-flow distortion generated by the nonlinear critical-layer interactions. The present analysis increases the cross-flow to an order of magnitude such that the buffer and critical layers coalesce. In this instance the nonlinear critical layer contains viscous as well as nonequilibrium effects. The resulting amplitude equations are solved for perturbations initiated at a fixed station in the flow.

\$1. Introduction. Vortex/wave interaction in which the oncoming boundary layer has a small amount of cross-flow, over a yawed wing for example, was discussed by Brown and Smith (1996) and the analysis was subsequently extended to jet type flows with small swirl by the present authors (Allen et al. 1996). Both these studies involve the non-linear interaction of two oblique inflexional Rayleigh waves, and are a development from those of the symmetric situations of Brown et al. (1993) and Smith, Brown and Brown (1993, referred to herein as SBB) to a pair of unequal amplitude input waves. Early papers in this field, which has attracted considerable attention recently in connection with theories of laminar/turbulent transition, are those of Hall and Smith $(1988,1989,1990,1991)$ and, in various flow and parameter regimes, those of Benney and Chow (1989), Goldstein and Leib (1989), Goldstein and Choi (1989), Wu (1992) and Wu, Lee and Cowley (1993). The last of these papers is particularly relevant to the present study and will be referenced here subsequently as WLC. 
Our ultimate aim is to study vortex/wave interactions with a cross flow that is of order unity in terms of the global Reynolds number $R$, assumed to be large. In Brown and Smith (1996) the cross-flow was represented as a perturbation $O\left(R^{-1 / 4}\right)$ to the flow in the main direction, that of $x$, and an adaptation of the SBB approach was appropriate. Two input waves, of differing amplitudes, made equal angles $\tan ^{-1} \beta / \alpha$ with the $x$-direction with the ratio $\beta / \alpha$ being $O(1)$. Here $\alpha, \beta$ are respectively the wave numbers in the $x$-direction and in the $z$-direction normal to it. In the present study the cross-flow perturbation is increased by an order of magnitude to an $O\left(R^{-1 / 6}\right)$ value, maintaining the arbitrary, order unity, angle made by the waves with the $x$-direction. The appropriate scales and flow regimes now correspond to those of WLC and we require the extension of their analysis to non-symmetric input as the presence of cross-flow precludes the existence of waves of equal amplitude. The resulting amplitude equations reduce to theirs and to those of Brown and Smith (1996) in appropriate limits.

Successful studies of an $O(1)$ cross-flow have been made. Notable examples are that of Tollmien-Schlichting, rather than Rayleigh wave input, by Davis and Smith (1994), and of the Rayleigh wave study of Gajjar (1995). The latter has a wave amplitude depending on two space variables but is restricted to the case of a small angle between the wave and the direction in which the oncoming profile is inflexional. In both this work, and Gajjar (1996) in which long wavelengths are examined (see also Bassom and Gajjar 1988), the mainstream speed at the critical layer is zero or small.

The plan of the present work is as follows. In $\$ 2$ the equations of motion and appropriate length scales are set out. In $\$ 3$ we present the solution in the core away from the critical level which occurs at an inflexional point of the basic profile, and the implications of the subsequent match with the critical layer are noted. In $\$ 4$ the equations in the neighbourhood of this level are given, and the required solutions at first and second order are obtained in $\$ \$ 5,6$. The study represents a fixed-frequency analysis, but involves a "nonequilibrium" critical layer in $x$ which here replaces the time co-ordinate of WLC. At each stage of the critical-layer expansion we have followed Hickernell (1984) and have solved an initial-value problem in $x$ to model a perturbation initiated at $x=0$. For clarity, and to keep track of the calculation, the solutions are presented in forms corresponding to those of WLC to which they reduce as the cross-flow parameter $\mu \rightarrow 0$, the amplitude functions become equal and certain regions of integration become unbounded.

The resulting coupled amplitude equations are discussed and numerical solutions presented in $\$ \$ 8,9$. They are more general than those of WLC in that equal amplitudes for the two waves are not possible now, but have very similar properties in that the terminating singularity, first proposed by Goldstein and Choi (1989) for an inviscid study corresponding to that of WLC, is again appropriate here, and has been encountered irrespective of the initial conditions. An "initial" rather than a terminal singularity proposed by Wu (1996, private communication), for a related situation in a parameter range in which Tollmien-Schlichting waves rather than Rayleigh waves are appropriate, cannot occur here. The paper concludes with further comments in $\$ 10$. 
\$2. The equations of motion and the length scales. The equations required are the incompressible Navier-Stokes equations non-dimensionalised with a suitable fundamental length and velocity in the form

$$
\nabla \cdot \underline{q}^{*}=0, \quad \frac{\partial \underline{q}^{*}}{\partial t^{*}}+\left(\underline{q}^{*} \cdot \nabla\right) \underline{q}^{*}=-\nabla p^{*}+R^{-1} \nabla^{2} \underline{q}^{*} .
$$

In (2.1), $q^{*}$ and $p^{*}$ are the velocity and pressure, $t^{*}$ is a non-dimensional time, and $R$ is the Reynolds number subsequently assumed to be large.

The physical situation under consideration is that of a classical three-dimensional laminar boundary layer, with flow quantities independent of one of the horizontal coordinates of which, to leading order in the inverse Reynolds number, the streamwise velocity profile develops an inflection point, at the station $x^{*}=0$ say, and the flow becomes neutrally stable. Further discussion of this point is made in $\$ 8$. Here $x^{*}, y^{*}, z^{*}$ are Cartesian coordinates with the wall at $y^{*}=0$, and $u^{*}, v^{*}, w^{*}$ are the corresponding velocity components.

We now define

$$
\varepsilon=R^{-1 / 12}
$$

where $\varepsilon \ll 1$ and describe the scales to be employed. The velocity components $u^{*}, v^{*}, w^{*}$ and pressure in (2.1) are written as

$$
\begin{aligned}
& \left(u^{*}, v^{*}, w^{*}, p^{*}\right) \\
& \quad=\left\{U\left(y, x^{*}\right)+u, \quad \varepsilon^{6} V\left(y, x^{*}\right)+v, \quad \varepsilon^{2} W\left(y, x^{*}\right)+w, \quad P\left(y, x^{*}\right)+p\right\}
\end{aligned}
$$

where $y=\varepsilon^{-6} y^{*}$ is the usual boundary-layer variable, and $U, \varepsilon^{6} V, \varepsilon^{2} W, P$ are the velocity components and pressure of the oncoming boundary layer, the cross-flow $\varepsilon^{2} W$ being evident here.

The ensuing analysis will be carried out on a fast time-scale, with two fast length-scales in the principle horizontal direction, a fast length scale in the transverse direction and the usual normal length-scales for a boundary layer involving a critical level. Thus

$$
t^{*}=\varepsilon^{6} T, \quad x^{*}=\varepsilon^{6} X=\varepsilon^{4} x, \quad z^{*}=\varepsilon^{6} z
$$

for the time and horizontal length-scales, and

$$
y^{*}=\varepsilon^{6} y=\varepsilon^{6}\left(y_{c}+\varepsilon^{2} Y\right)
$$

for the normal length-scales. In (2.5), $y_{c}$ is the value of $y$ at the critical level where the horizontal velocity component develops its point of inflection so that $U_{0}^{\prime \prime}\left(y_{c}\right)=0$ where $U_{0}(y)=U(y, 0)$.

There are two regions in which the solution of (2.1) is to be obtained, namely the core flow in which $y=O(1)$ and the neighbourhood of the critical layer in which $Y=O(1)$. In the following section we consider the first of these.

\$3. The core flow and the solvability condition. In the core away from the critical layer we write

$$
u=\varepsilon^{6} u_{1}+\varepsilon^{8} u_{2}+\ldots
$$


with corresponding expressions for $v, w$ and $p$ in (2.3). To the order of approximation required in (3.1) the viscous terms in (2.1) are not necessary and $U, V, W$ in (2.3) may be replaced by their values, $U_{0}(y), V_{0}(y), W_{0}(y)$ at $x^{*}=$ 0 . Comments on the absence of the effect of the non-parallelism of the basic flow are made in $\$ 8$. We also define

$$
E=e^{i \alpha\left(X-c_{0} T\right)}
$$

where $\alpha(>0)$ is the constant wave number in the $x^{*}$ direction and $c_{0}$ is the value of $U_{0}(y)$ at the critical layer so that $U_{0}\left(y_{c}\right)=c_{0}$. The faster length and time scales are confined to $E$ and in addition we write

$$
v_{1}=\left(A_{+}(x) e^{i \beta z}+A_{-}(x) e^{-i \beta z}\right) E v_{1}^{(1)}(y)+\text { c.c. }
$$

where the constant $\beta$ is the transverse wave number and c.c. denotes the complex conjugate. The purpose of this study is to obtain equations satisfied by the amplitude $A_{ \pm}(x)$. As usual, $v_{1}^{(1)}$ satisfies Rayleigh's equation

$$
\left(u_{0}-c_{0}\right)\left(D^{2}-\bar{\alpha}^{2}\right) v_{1}^{(1)}-U_{0}^{\prime \prime} v_{1}^{(1)}=0, \quad D=d / d y,
$$

with $v_{1}^{(1)}(0)=v_{1}^{(1)}(\infty)=0$ and $\bar{\alpha}^{2}=\alpha^{2}+\beta^{2}$. If the streamwise wavenumber $\alpha$ in (3.2) is prescribed then $\beta$ in (3.4) is determined as the eigenvalue of the Rayleigh problem.

The quantities $u_{1}, w_{1}$ and $p_{1}$ are found as

$$
\begin{aligned}
& u_{1}=\frac{i}{\alpha \bar{\alpha}^{2}}\left(\alpha^{2} \frac{\partial v_{1}}{\partial y}+\beta^{2} \frac{U_{0}^{\prime} v_{1}}{U_{0}-c_{0}}\right)+u_{1}^{(0,2)} e^{2 i \beta z}+u_{1}^{(0,-2)} e^{-2 i \beta z}+\text { c.c. } \\
& w_{1}=\frac{i \beta}{\bar{\alpha}^{2}}\left(D v_{1}^{(1)}-\frac{U_{0}^{\prime}}{U_{0}-c_{0}} v_{1}^{(1)}\right)\left(A_{+} e^{i \beta z}-A_{-} e^{-i \beta z}\right) E+\text { c.c. } \\
& p_{1}=\frac{i \alpha}{\bar{\alpha}^{2}}\left(U_{0}^{\prime} v_{1}-\left(U_{0}-c_{0}\right) \frac{\partial v_{1}}{\partial y}\right)+\text { c.c., }
\end{aligned}
$$

where in (3.5a) the $E$-independent vortex terms in $\exp ( \pm 2 i \beta z)$ are forced by the critical layer as will be seen in $\$ 4$ below.

For $v_{2}$ we write, since it is also found to contain a vortex contribution forced by the critical layer,

$v_{2}=\left(v_{2}^{(1,1)}(y, x) e^{i \beta z}+v_{2}^{(1,-1)}(y, x) e^{-i \beta z}\right) E+v_{2}^{(0,2)} e^{2 i \beta z}+v_{2}^{(0,-2)} e^{-2 i \beta z}+$ c.c.

and find that $v_{2}^{(1,1)}$ satisfies

$$
\begin{aligned}
& \left(D^{2}-\bar{\alpha}^{2}\right) v_{2}^{(1,1)}-\left\{U_{0}^{\prime \prime} v_{2}^{(1,1)} /\left(U_{0}-c\right)\right\} \\
& \quad=v_{1}^{(1)}\left[A_{+}^{\prime}(x)\left(\frac{i c_{0} U^{\prime \prime}}{\alpha\left(U_{0}-c_{0}\right)^{2}}-2 i \alpha\right)+\frac{\beta}{\alpha} \frac{A_{+}(x)}{U_{0}-c_{0}}\left(W_{0}^{\prime \prime}-\frac{W_{0} U_{0}^{\prime}}{U_{0}-c_{0}}\right)\right]
\end{aligned}
$$

subject to $v_{2}^{(1,1)}(0)=v_{2}^{(1,1)}(\infty)=0$. The equation for $v_{2}^{(1,-1)}$ is obtained by replacing $A_{+}$by $A_{-}$and $\beta$ by $-\beta$ in (3.7).

Since $U_{0}^{\prime \prime}\left(y_{c}\right)=0$ the solution $v_{1}^{(1)}$ of (3.4) is regular at $y=y_{c}$ with $v_{1}^{(1)}\left(y_{c}\right)=1$ say. We shall see that a match with the critical layer of $\$ 4$ requires 
$v_{2}^{(1,1)}$ to be continuous at $y=y_{c}$ but that as $y \rightarrow y_{c} \pm 0$ the derivative

$$
\frac{\partial v_{2}^{(1,1)}}{\partial y} \approx f(x) \log \left|y-y_{c}\right|+J_{ \pm}^{(1,1)}
$$

where, by reduction of the order of equation (3.7) or otherwise,

$$
f(x)=\frac{i c_{0} U_{c}^{\prime \prime \prime}}{\alpha U_{c}^{\prime 2}} A_{+}^{\prime}(x)+\frac{\beta}{\alpha}\left(\frac{W_{c}^{\prime \prime}}{U_{c}^{\prime}}-\frac{W_{c} U_{c}^{\prime \prime \prime}}{U_{c}^{\prime 2}}\right) A_{+}(x),
$$

and

$$
\begin{aligned}
J_{+}^{(1,1)}-J_{-}^{(1,1)}=-\frac{i}{\alpha} A_{+}^{\prime}(x) & \int_{0}^{\infty}\left(\frac{c_{0} U_{0}^{\prime \prime}}{\left(U_{0}-c_{0}\right)^{2}}-2 \alpha^{2}\right)\left(v_{1}^{(1)}\right)^{2} d y \\
& -\frac{\beta}{\alpha} A_{+}(x) \int_{0}^{\infty}\left(W_{0}^{\prime \prime}-\frac{W_{0} U_{0}^{\prime \prime}}{U_{0}-c_{0}}\right) \frac{\left(v_{1}^{(1)}\right)^{2} d y}{U_{0}-c_{0}} .
\end{aligned}
$$

In (3.9), $U_{c}^{\prime}=U_{0}^{\prime}\left(y_{c}\right)$ etc. and in (3.10) the integrals are to be interpreted as Cauchy principal values. When the jump in the derivative in (3.8) is matched to the appropriate term in the critical layer the required amplitude equation is obtained.

The argument of this section, including the insertion of the vortex terms into (3.5a) and (3.6), is exactly analogous to that of WLC although those authors allowed for more than one critical layer for each of which the corresponding $v_{1}^{(1,1)}$ could be singular. To simplify the calculation they took $A_{+}(x)=$ $A_{-}(x)$ and consequently $v_{1}^{(1,1)}=v_{1}^{(1,-1)}$. As can be seen from (3.7) the presence of the cross-flow $W_{0}$ means that this is not possible here and consequently $A_{ \pm}$ must both be retained.

At first sight it might seem that the cross-flow effects could be eliminated by a rotation of axes. However this is not so as may be appreciated as follows. The basic flow is, until the vortex of order $\varepsilon^{6}$ intrudes in $u_{1}, z$-independent. The $x^{*}$ direction is thus determined and it is the velocity component in this direction that is assumed to have an inflection point at $x^{*}=0$ leading to a nontrivial solution of Rayleigh's equation. The waves, of amplitude $A_{ \pm}$respectively, make equal and opposite $O(1)$ angles $\tan ^{-1} \beta / \alpha$ with this direction, while the mainstream makes an angle $O\left(\varepsilon^{2}\right)$ with it. This is in contrast with the work of Gajjar (1995) in which the angle between the waves and the inflection direction is small although the cross-flow is $O(1)$. In Gajjar (1996) both wavelengths are taken to be large.

It is clear from (3.5) and (3.8) that it is necessary to consider the neighbourhood of the critical layer where $y=y_{c}$ and $U_{0}\left(y_{c}\right)=c_{0}$. The solution is formulated in the following section.

\$4. The relevant equations in the neighbourhood of the critical layer. To discuss the neighbourhood of the critical layer we require the variable $Y$ defined in $(2.5)$ by

$$
y=y_{c}+\varepsilon^{2} Y,
$$


and write, for $u, v, w, p$ in $(2.3)$

$$
\begin{aligned}
& u=\varepsilon^{4} U_{1}+\varepsilon^{6} U_{2}+\varepsilon^{8} U_{3}+\ldots, \\
& v=\varepsilon^{6} V_{1}+\varepsilon^{8} V_{2}+\varepsilon^{10} V_{3}+\ldots, \\
& w=\varepsilon^{4} W_{1}+\varepsilon^{6} W_{2}+\varepsilon^{8} W_{3}+\ldots, \\
& p=\varepsilon^{6} P_{1}+\varepsilon^{8} P_{2}+\varepsilon^{10} P_{3}+\ldots
\end{aligned}
$$

The poles in $(3.5 \mathrm{a}, \mathrm{b})$ in $u_{1}, w_{3}$ at $y=y_{c}$ lead to the increased orders of magnitude of $u, w$ in this layer. The functions $U, V, W, P$ in (2.3) are expanded near $x^{*}=0$ and $y=y_{c}$ in the form

$$
\begin{aligned}
& U\left(y, x^{*}\right)=c_{0}+\varepsilon^{2} b_{1} Y+\varepsilon^{4} c_{1} x+\varepsilon^{6}\left(d_{1} x Y+\frac{1}{6} b_{3} Y^{3}\right) \\
&+\varepsilon^{8}\left(c_{2} x^{2}+\frac{1}{2} d_{2} x Y^{2}+\frac{1}{24} b_{4} Y^{4}\right)+\ldots \\
& V\left(y, x^{*}\right)=r_{0}-\varepsilon^{2} c_{1} Y+\varepsilon^{4}\left(r_{1} x-\frac{1}{2} d_{1} Y^{2}\right)+\ldots \\
& W\left(y, x^{*}\right)=g_{0}+\varepsilon^{2} g_{1} Y+\varepsilon^{4}\left(h_{0} x+\frac{1}{2} g_{2} Y^{2}\right) \\
&+\varepsilon^{6}\left(h_{1} x Y+\frac{1}{6} g_{3} Y^{3}\right)+\ldots \\
& P\left(y, x^{*}\right)=q_{0}+\varepsilon^{4} q_{1} x+\varepsilon^{8} q_{2} x^{2}+\ldots
\end{aligned}
$$

in which all the coefficients are constants determined by the non-interactive oncoming boundary layer. Terms $O\left(\varepsilon^{8} \log \varepsilon\right)$ have been omitted from $(4.2 \mathrm{a}, \mathrm{c})$ and $O\left(\varepsilon^{10} \log \varepsilon\right)$ from $(4.2 \mathrm{~b}, \mathrm{~d})$ as these terms automatically match to the core flow and have no effect on the amplitude equations.

The equations for $U_{j}, V_{j}, W_{j}, P_{j}$ in (4.2) are obtained from (2.1) to (2.5) as follows.

(a) The continuity equation. Equation (2.1a) leads to

$$
\begin{gathered}
C\left(U_{1}, V_{1}, W_{1}\right)=0, \\
C\left(U_{j}, V_{j}, W_{j}\right)=-\partial U_{j-1} / \partial x, \quad j=2,3,
\end{gathered}
$$

where

$$
C(U, V, W) \equiv \frac{\partial U}{\partial X}+\frac{\partial V}{\partial Y}+\frac{\partial W}{\partial z}
$$

(b) The $x$-momentum equation. The first component of equation (2.1b) gives

$$
\begin{aligned}
M_{X}\left(U_{1}, V_{1}, P_{1}\right)= & 0, \\
M_{X}\left(U_{2}, V_{2}, P_{2}\right)=-\left(U_{1} \frac{\partial U_{1}}{\partial X}+V_{1} \frac{\partial U_{1}}{\partial Y}+W_{1} \frac{\partial U_{1}}{\partial z}\right)-\frac{\partial P_{1}}{\partial x} & \\
& -x c_{1} \frac{\partial U_{1}}{\partial X}-b_{1} Y \frac{\partial U_{1}}{\partial x}-r_{0} \frac{\partial U_{1}}{\partial Y}-g_{1} Y \frac{\partial U_{1}}{\partial z},
\end{aligned}
$$




$$
\begin{aligned}
M_{X}\left(U_{3}, V_{3}, P_{3}\right) & =-\left(U_{1} \frac{\partial U_{2}}{\partial X}+U_{2} \frac{\partial U_{1}}{\partial X}+V_{1} \frac{\partial U_{2}}{\partial Y}+V_{2} \frac{\partial U_{1}}{\partial Y}\right. \\
& \left.+W_{1} \frac{\partial U_{2}}{\partial z}+W_{2} \frac{\partial U_{1}}{\partial z}+U_{1} \frac{\partial U_{1}}{\partial x}\right)-\frac{\partial P_{2}}{\partial x_{1}} \\
& -x c_{1} \frac{\partial U_{2}}{\partial X}-\left(d_{1} x Y+\frac{1}{6} b_{3} Y^{3}\right) \frac{\partial U_{1}}{\partial X}-b_{1} Y \frac{\partial U_{2}}{\partial x} \\
& -c_{1} x \frac{\partial U_{1}}{\partial x}-c_{1} U_{1}-r_{0} \frac{\partial U_{2}}{\partial Y}+c_{1} Y \frac{\partial U_{1}}{\partial Y}-\left(d_{1} x+\frac{1}{2} b_{3} Y^{2}\right) V_{1} \\
& -g_{1} Y \frac{\partial U_{2}}{\partial z}-\left(h_{0} x+\frac{1}{2} g_{2} Y^{2}\right) \frac{\partial U_{1}}{\partial z}+\lambda\left(\frac{\partial^{2} U_{1}}{\partial X^{2}}+\frac{\partial^{2} U_{1}}{\partial z^{2}}\right)
\end{aligned}
$$

where

$$
M_{X}(U, V, P) \equiv b_{1} Y \frac{\partial U}{\partial X}+c_{0} \frac{\partial U}{\partial x}+g_{0} \frac{\partial U}{\partial z}+b_{1} V+\frac{\partial P}{\partial X}-\lambda \frac{\partial^{2} U}{\partial Y^{2}}
$$

Here, following WLC, we have multiplied the viscous terms by a book-keeping parameter $\lambda$ so that the inviscid limit $\lambda=0$ can be readily identified.

(c) The $y$-momentum equation. The second component of equation (2.1b) gives

$$
\begin{gathered}
\frac{\partial P_{1}}{\partial Y}=0, \quad \frac{\partial P_{2}}{\partial Y}=0 \\
\frac{\partial P_{3}}{\partial Y}=-b_{1} Y \frac{\partial V_{1}}{\partial X}-c_{0} \frac{\partial V_{1}}{\partial x}-g_{0} \frac{\partial V_{1}}{\partial z} .
\end{gathered}
$$

(d) The $z$-momentum equation. The third component of equation (2.1a) gives

$$
\begin{aligned}
M_{z}\left(W_{1}, P_{1}\right)= & , \\
M_{z}\left(W_{2}, P_{2}\right)= & -\left(U_{1} \frac{\partial W_{1}}{\partial X}+V_{1} \frac{\partial W_{1}}{\partial Y}+W_{1} \frac{\partial W_{1}}{\partial z}\right) \\
& -x c_{1} \frac{\partial W_{1}}{\partial X}-b_{1} Y \frac{\partial W_{1}}{\partial x}-r_{0} \frac{\partial W_{1}}{\partial Y}-g_{1} V_{1}-g_{1} Y \frac{\partial W_{1}}{\partial z}, \\
M_{z}\left(W_{3}, P_{3}\right)= & -\left(U_{1} \frac{\partial W_{2}}{\partial X}+U_{2} \frac{\partial W_{1}}{\partial X}+V_{1} \frac{\partial W_{2}}{\partial Y}+V_{2} \frac{\partial W_{1}}{\partial Y}+W_{1} \frac{\partial W_{2}}{\partial z}+W_{2} \frac{\partial W_{1}}{\partial z}\right) \\
& -x c_{1} \frac{\partial W_{2}}{\partial X}-\left(d_{1} x Y+\frac{1}{6} b_{3} Y^{3}\right) \frac{\partial W_{1}}{\partial X}-b_{1} Y \frac{\partial W_{2}}{\partial x} \\
& -c_{1} x \frac{\partial W_{1}}{\partial x}-r_{0} \frac{\partial W_{2}}{\partial Y}+c_{1} Y \frac{\partial W_{1}}{\partial Y}-g_{1} V_{2}-g_{2} Y V_{1}-g_{1} Y \frac{\partial W_{2}}{\partial z} \\
& -\left(h_{0} x+\frac{1}{2} g_{2} Y^{2}\right) \frac{\partial W_{1}}{\partial z}+\lambda\left(\frac{\partial^{2} W_{1}}{\partial X^{2}}+\frac{\partial^{2} W_{1}}{\partial z^{2}}\right),
\end{aligned}
$$


where

$$
M_{z}(W, P) \equiv b_{1} Y \frac{\partial W}{\partial X}+c_{0} \frac{\partial W}{\partial x}+g_{0} \frac{\partial W}{\partial z}+\frac{\partial P}{\partial z}-\lambda \frac{\partial^{2} W}{\partial z^{2}}
$$

Henceforth we shall ignore the linear forcing on the right-hand sides of equations (4.4), (4.6), (4.8) and (4.9). As usual, see for example WLC, SBB or any of the earlier examples of linear critical layers, whether viscous or nonequilibrium or, as here, a combination of both, the effect of these terms on the jump $J_{+}-J_{-}$in (3.10) is to increase the logarithm in (3.8) by $i \pi \operatorname{sgn} b_{1}$ as $y$ goes from $y_{c}-0$ to $y_{c}+0$. Thus the linear forcing terms in the equations of this section give a contribution to $J_{+}-J_{-}$of

$$
\left(J_{+}^{(1,1)}-J_{-}^{(1,1)}\right)_{L}=i \pi f(x) \operatorname{sgn} b_{1}
$$

where $f(x)$ is defined in (3.9). This must be added to the non-linear contribution in the conclusion.

The following section contains the solutions for the leading terms $U_{1}, V_{1}, W_{1}, P_{1}$.

\$5. The leading-order solutions in the critical layer. Since, with a few unimportant exceptions, our solution will reduce to that of WLC when $g_{0}=0$ and $A_{+}=A_{-}$, i.e., when the cross-flow is zero and the two waves in (3.3) have equal amplitude, we use their notation when convenient. As there it follows from (4.4a), (4.6a), (4.8a), that

$$
\begin{aligned}
& V_{1}=\left(A_{+}(x) e^{i \beta_{z}}+A_{-}(x) e^{-i \beta_{z}}\right) E+\text { c.c. } \\
& P_{1}=\frac{i \alpha}{\bar{\alpha}^{2}} V_{1}
\end{aligned}
$$

on use of (3.5c). Equation (4.9a) may be written as

$$
L_{0} W_{1}=-\frac{\partial P_{1}}{\partial z}
$$

where, by definition,

$$
L_{0} \equiv c_{0} \frac{\partial}{\partial x}+b_{1} Y \frac{\partial}{\partial X}+g_{0} \frac{\partial}{\partial z}-\lambda \frac{\partial^{2}}{\partial Y^{2}} .
$$

If we write

$$
W_{1}=\left(W_{1}^{(1,1)} e^{i \beta z}+W_{1}^{(1,-1)} e^{-i \beta z}\right) E+\text { c.c. }
$$

and define

$$
\Omega=\alpha b_{1} Y / c_{0}, \quad s=\lambda b_{1}^{2} \alpha^{2} /\left(3 c_{0}^{3}\right), \quad \mu=\beta g_{0} / c_{0}
$$


then the solution of (5.3) for $W_{1}^{(1,1)}$ is

$$
W_{1}^{(1,1)}=\frac{\beta \alpha b_{1}}{c_{0} \bar{\alpha}^{2}} \int_{0}^{\infty} A_{+}(x-\xi) e^{-i(\Omega+\mu) \xi-s \xi^{\xi}} d \xi .
$$

To obtain $W_{1}^{(1,-1)}$ it is necessary to replace $A_{+}$by $A_{-}, \beta$ by $-\beta$ and $\mu$ by $-\mu$. Finally, if

$$
U_{1}=\left(U_{1}^{(1,1)} e^{i \beta z}+U_{1}^{(1,-1)} e^{-i \beta z}\right) E+\text { c.c. }
$$

then it follows from the continuity equation (4.4a) that

$$
U_{1}^{(1, \pm 1)}=\mp \frac{\beta}{\alpha} W_{1}^{(1, \pm 1)}
$$

The form of the solution (5.7) is that used by WLC and is relevant when $A_{+}(x) \rightarrow 0$ as $x \rightarrow-\infty$. Indeed in the inviscid limit $s=0$, this condition is necessary for the integral to converge. We prefer here to focus attention on a perturbation that is initiated at $x=0$ with $A(0)$ prescribed and $U_{1}, W_{1}$, initially zero. To satisfy this condition, the upper limit in the integral in (5.7) must be replaced by $x$. With this change equation (5.3) is still satisfied but, from (5.7), for $|Y| \gg 1$,

$$
W_{1}^{(1,1)} \approx-\frac{i \beta}{\bar{\alpha}^{2} Y}\left[A_{+}(x)-A_{+}(0) e^{-s x^{3}} e^{-i(\Omega+\mu) x}\right] .
$$

The first of these terms is exactly as required to match with the pole predicted by $(3.5 \mathrm{~b})$. The second term is analogous to that encountered by Hickernell (1984) in his discussion of time-dependent critical layers in shear flows on the beta plane. It can be shown to match to an eigensolution in the core and has no effect on the amplitude equation for $A_{ \pm}(x)$. A similar conclusion was reached by Hickernell who described it as a transient of the initial value problem. Further discussion of this point is deferred to $\$ 8$.

\$6. The solutions at the second order. At the second order the terms with subscript 2 in (4.2) take the form

$$
\begin{aligned}
V_{2}= & V_{2}^{(0.2)} e^{2 i \beta z}+V_{2}^{(0,-2)} e^{-2 i \beta z}+V_{2}^{(2,0)} E^{2}+\text { c.c. } \\
U_{2}= & U_{2}^{(0,2)} e^{2 i \beta z}+U_{2}^{(0,-2)} e^{-2 i \beta z}+U_{2}^{(0,0)} \\
& +\left(U_{2}^{(2,2)} e^{2 i \beta z}+U^{(2,-2)} e^{-2 i \beta z}+U_{2}^{(2,0)}\right) E^{2}+\text { c.c. } \\
W_{2}= & W_{2}^{(0,2)} e^{2 i \beta z}+W_{2}^{(0,-2)} e^{-2 i \beta z}+W_{2}^{(0,0)} \\
& +\left(W_{2}^{(2,2)} e^{2 i \beta z}+W^{(2,-2)} e^{-2 i \beta z}+W_{2}^{(2,0)}\right) E^{2}+\text { c.c. },
\end{aligned}
$$

where the lack of symmetry $\left(A_{+} \neq A_{-}\right)$leads to the introduction of the two $z_{-}$ independent terms in (6.1c). From (4.8b), $P_{2}$ is independent of $Y$ and may be calculated from the outer solution if required. First harmonic, $O(E)$, contributions to (6.1) will not subsequently be required. 
For convenience we now define

$$
\chi_{n}^{ \pm}=\int_{0}^{x} \xi^{n} A_{ \pm}(x-\xi) e^{-i(\Omega \pm \mu) \xi-s \xi^{3}} d \xi
$$

so that for example, in (5.7) with the upper limit of integration replaced by $x$, $W_{1}^{(1,1)}=\left(\beta \alpha b_{1} / c_{0} \bar{\alpha}^{2}\right) \chi_{0}^{+}$.

(a) The solutions for $V_{2}$. As in WLC equations may be found for the components of $\partial^{2} V_{2} / \partial Y^{2}$. From (4.4b), (4.6b), (4.8b), (4.9b) these are found to be

$$
\begin{gathered}
\left(c_{0} \frac{\partial}{\partial x}+2 i \beta g_{0}-\frac{\lambda \hat{\sigma}^{2}}{\partial Y^{2}}\right) \frac{\partial^{2} V_{2}^{(0,2)}}{\partial Y^{2}}=-\frac{2 i \beta^{2} \alpha^{3} b_{1}^{3}}{\bar{\alpha}^{2} c_{0}^{3}}\left[\tilde{A}_{-} \chi_{2}^{+}+\frac{4 \beta^{2}}{\bar{\alpha}^{2}} \tilde{\chi}_{0}^{-} \chi_{1}^{+}\right] \\
\left(c_{0} \frac{\partial}{\partial x}+2 i \alpha b_{1} Y-\frac{\lambda \partial^{2}}{\partial Y^{2}}\right) \frac{\partial^{2} V_{2}^{(2,0)}}{\partial Y^{2}} \\
=\frac{2 i \beta^{2} \alpha^{3} b_{1}^{3}}{\bar{\alpha}^{2} c_{0}^{3}}\left[A+\chi_{2}^{-}+A-\chi_{2}^{+}+\frac{4 \beta^{2}}{\bar{\alpha}^{2}}\left(\chi_{1}^{+} \chi_{0}^{-}+\chi_{0}^{+} \chi_{1}^{-}\right)\right],
\end{gathered}
$$

with the equation for $V_{2}^{(0,-2)}$ to be obtained by interchanging the plus and minus signs, in the sub and superscripts on the right-hand side of (6.3). Also in (6.3), and subsequently, the tilde denotes complex conjugation.

The solutions of (6.3), (6.4) are

$$
\begin{aligned}
& \frac{\partial^{2} V_{2}^{(0,2)}}{\partial Y^{2}}=-\frac{2 i \beta^{2} \alpha^{3} b_{1}^{3}}{\bar{\alpha}^{2} c_{0}^{4}} \iint_{\Delta} \xi I_{v}^{(0,2)}(\xi, \eta) e^{-i \Omega \xi} e^{-i \mu(\xi+2 \eta)} \\
& \quad \times \tilde{A}_{-}(x-\eta) A_{+}(x-\eta-\xi) d \xi d \eta, \\
& \begin{aligned}
\frac{\partial^{2} V_{2}^{(2,0)}}{\partial Y^{2}}=\frac{2 i \beta^{2} \alpha^{3} b_{1}^{3}}{\bar{\alpha}^{2} c_{0}^{4}} \iint_{\Delta} I_{v}^{(2,0)}(\xi, \eta) e^{-i \Omega(\xi+2 \eta)} \\
\quad \times\left[e^{-i \mu \xi} A_{-}(x-\eta) A_{+}(x-\eta-\xi)\right. \\
\left.+e^{i \mu \xi} A_{+}(x-\eta) A_{-}(x-\eta-\xi)\right] d \xi d \eta,
\end{aligned}
\end{aligned}
$$

where, as in WLC equations (3.36) to (3.39),

$$
\begin{aligned}
I_{v}^{(0,2)}(\xi, \eta) & =I^{(0)}(\xi, \eta)\left[\xi+\frac{4 \beta^{2}}{\bar{\alpha}^{2}} \int_{0}^{\eta} e^{-s(2 \zeta+3 \xi) \zeta^{2}} d \zeta\right] \\
I_{v}^{(2,0)}(\xi, \eta) & =I^{(1)}(\xi, \eta)\left[\xi^{2}+\frac{4 \beta^{2}}{\bar{\alpha}^{2}} \int_{0}^{\eta}(\xi+2 \zeta) e^{s(2 \zeta+3 \xi) \zeta^{2}} d \zeta\right] \\
I^{(0)}(\xi, \eta) & =e^{-s\left(\xi^{3}+3 \xi^{2} \eta\right)} \\
I^{(1)}(\xi, \eta) & =e^{-s\left(\xi^{3}+3 \xi^{2} \eta+6 \xi \eta^{2}+4 \eta^{3}\right)}
\end{aligned}
$$


The solution for $\partial^{2} V^{(0,-2)} / \partial Y^{2}$ may be written down by analogy with (6.5). In equations (6.5) and (6.6) the region of integration in the $\xi \eta$-plane is denoted by $\Delta$. In the case of the initial value problem this is the triangle in the positive quadrant over which the arguments of the unknown amplitudes in the integrals are positive, namely $0 \leqslant \xi+\eta \leqslant x$. If decay as $x \rightarrow-\infty$ is to be prescribed then the region of integration is the whole positive quadrant.

(b) The solutions for $W_{2}$. The equations for the mean-flow distortion $W_{2}^{(0,0)}$ may be written as

$$
\left(c_{0} \frac{\partial}{\partial x}-\frac{\lambda \partial^{2}}{\partial Y^{2}}\right) W_{2}^{(0,0)}=\frac{i \beta \alpha^{2} b_{1}^{2}}{\bar{\alpha}^{2} c_{0}^{2}}\left[\tilde{A}_{+} \chi_{1}^{+}-\tilde{A}_{-} \chi_{1}^{-}\right],
$$

while $W_{2}^{(0,2)}$ may be obtained from the continuity equation as

$$
W_{2}^{(0,2)}=\frac{i}{2 \beta} \frac{\partial V_{2}^{(0.2)}}{\partial Y}
$$

The solution for $W_{2}^{(0,0)}$, which is zero in WLC since the right-hand side of (6.10) vanishes when $A_{+}=A_{-}$and $\mu=0$, is

$$
\begin{aligned}
W_{2}^{(0,0)}= & \frac{i \beta \alpha^{2} b_{1}^{2}}{\bar{\alpha}^{2} c_{0}^{3}} \iint_{\Lambda} \xi I^{(0)}(\xi, \eta) e^{-i \Omega \xi} \\
& \times\left[e^{-i \mu \xi} \tilde{A}_{+}(x-\eta) A_{+}(x-\eta-\xi)\right. \\
& \left.-e^{i \mu \xi} \tilde{A}_{-}(x-\eta) A_{-}(x-\eta-\xi)\right] d \xi d \eta .
\end{aligned}
$$

For $W_{2}^{(2,2)}$ the equation to be satisfied is

$$
\left(c_{0} \frac{\partial}{\partial x}+2 i \alpha b_{1} Y+2 i \beta g_{0}-\frac{\lambda \partial^{2}}{\partial Y^{2}}\right) W_{2}^{(2,2)}=\frac{i \beta \alpha^{2} b_{1}^{2}}{\bar{\alpha}^{2} c_{0}^{2}} A_{+} \chi_{1}^{+}
$$

the solution of which is

$$
\begin{aligned}
W_{2}^{(2,2)}=\frac{i \beta \alpha^{2} b_{1}^{2}}{\bar{\alpha}^{2} c_{0}^{3}} \iint_{\Delta} \xi I^{(1)}(\xi, & \eta) e^{-i \Omega(\xi+2 \eta)} e^{-i \mu(\xi+2 \eta)} \\
& \times A_{+}(x-\eta) A_{+}(x-\eta-\xi) d \xi d \eta .
\end{aligned}
$$

Finally the equation for $W_{2}^{(2,0)}$ in $(6.1 \mathrm{c})$ is

$$
\left(c_{0} \frac{\partial}{\partial x}+2 i \alpha b_{1} Y-\frac{\lambda \partial^{2}}{\partial Y^{2}}\right) W_{2}^{(2,0)}=-\frac{i \beta \alpha^{2} b_{1}^{2}}{\bar{\alpha}^{2} c_{0}^{2}}\left[A_{+} \chi_{1}^{-}-A_{-} \chi_{1}^{+}\right]
$$

with solution

$$
\begin{aligned}
W_{2}^{(2,0)}= & -\frac{i \beta \alpha^{2} b_{1}^{2}}{\vec{\alpha}^{2} c_{0}^{3}} \iint_{\Delta} \xi I^{(1)}(\xi, \eta) e^{-2 i \Omega 2 \eta} \\
& \times\left[e^{i \mu \xi} A_{+}(x-\eta) A_{-}(x-\eta-\xi)\right. \\
& \left.-e^{-i \mu \xi} A_{-}(x-\eta) A_{+}(x-\eta-\xi)\right] d \xi d \eta
\end{aligned}
$$


(c) The solutions for $U_{2}$. The equations for the mean-flow distortions $U_{2}^{(0,0)}$ and $U_{2}^{(0,2)}$ may be written as

$$
\begin{aligned}
\left(c_{0} \frac{\partial}{\partial x}-\frac{\lambda \partial^{2}}{\partial Y^{2}}\right) U_{2}^{(0,0)} & =-\frac{i \beta^{2} \alpha b_{1}^{2}}{\bar{\alpha}^{2} c_{0}^{2}}\left[\tilde{A}_{-} \chi_{1}^{-}+\tilde{A}_{+} \chi_{1}^{+}\right], \\
\left(c_{0} \frac{\partial}{\partial x}+2 i \beta g_{0}-\frac{\lambda \partial^{2}}{\partial Y^{2}}\right) U_{2}^{(0,2)} & =\frac{i \beta^{2} \alpha b_{1}^{2}}{\bar{\alpha}^{2} \eta c_{0}^{2}} A_{+} \tilde{\chi}_{1}^{-}-b_{1} V_{2}^{(0,2)}
\end{aligned}
$$

the solutions of which are

$$
\begin{aligned}
& \frac{\partial U_{2}^{(0,0)}}{\partial Y}=-\frac{\beta^{2} \alpha^{2} b_{1}^{3}}{\bar{\alpha}^{2} c_{0}^{4}} \iint_{\Delta} \xi^{2} I^{(0)}(\xi, \eta) e^{-i \Omega \xi}\left[e^{i \mu \xi} \tilde{A}_{-}(x-\eta) A_{-}(x-\eta-\xi) \eta\right. \\
& \left.+e^{-i \mu \xi} \tilde{A}_{+}(x-\eta) A_{+}(x-\eta-\xi)\right] d \xi d \eta, \\
& \frac{\partial U_{2}^{(0,2)}}{\partial Y}=-\frac{\beta^{2} \alpha^{2} b_{1}^{3}}{\bar{\alpha}^{2} c_{0}^{4}} \iint_{\Delta} I_{u}^{(0,2)}(\xi, \eta) e^{-i \Omega \xi} \\
& \times e^{-i \mu(2 \eta+\xi)} \tilde{A}_{-}(x-\eta) A_{+}(x-\eta-\xi) d \xi d \eta .
\end{aligned}
$$

In (6.20), $I_{u}^{(0,2)}(\xi, \eta)$ is the function defined in (3.48) of WLC, namely

$$
I_{u}^{(0,2)}(\xi, \eta)=I^{(0)}(\xi, \eta)\left[\xi^{2}+2 \xi \eta+\frac{4 \beta^{2}}{\bar{\alpha}^{2}} \int_{0}^{\eta} 2(\eta-\zeta) e^{-x(2 \zeta+3 \xi) \zeta^{2}} d \zeta\right] .
$$

The continuity equation yields the remaining coefficients in $(6.1 \mathrm{~b})$ as

$$
\begin{aligned}
U_{2}^{(2,0)} & =\frac{i}{2 \alpha} \frac{\partial V_{2}^{(2,0)}}{\partial Y}, \\
U_{2}^{(2, \pm 2)} & =\mp(\beta / \alpha) W_{2}^{(2, \pm 2)} .
\end{aligned}
$$

The vortex terms with exponents $(0,2)$ in $(3.5 \mathrm{a})$ and $(3.6)$ are induced by the critical layer as may be verified on examination of (6.5) and (6.20). From (6.5) it follows that

$$
\begin{aligned}
& {\left[V_{2}^{(0,2)}+\tilde{V}_{2}^{(0,-2)}\right]_{Y=-\infty}^{\infty}} \\
& \quad=\frac{16 \pi \beta^{4} \alpha b_{1}}{\vec{\alpha}^{4} c_{0}^{2}} \int_{\eta=0}^{x} \int_{\zeta=0}^{\eta} e^{-2 s \zeta^{3}} e^{-2 i \mu \eta} A_{+}(x-\eta) \tilde{A}_{-}(x-\eta) d \zeta d \eta
\end{aligned}
$$

and from $(6.20)$ that

$$
\begin{aligned}
{\left[U_{2}^{(0,2)}+\tilde{U}_{2}^{(0,-2)}\right]_{Y=-\infty}^{\infty}=-\frac{16 \pi \beta^{4} \alpha b_{1}^{2}}{\bar{\alpha}^{4} c_{0}^{3}} \int_{\eta=0}^{x} \int_{\zeta=0}^{\eta}(\eta-\zeta) e^{-2 s \zeta^{3}} e^{-2 i \mu \eta} } \\
\times A_{+}(x-\eta) \tilde{A}_{-}(x-\eta) d \zeta d \eta
\end{aligned}
$$


The quantities $U_{2}, V_{2}, W_{2}$ of this section together with $U_{1}, V_{1}, W_{1}$ of $\$ 5$ enable us to determine the amplitude equation for the unknown functions $A_{ \pm}$. This task is undertaken in the following section where the equation for $\partial^{2} V_{3}^{(1.1)} / \partial Y^{2}$ is considered.

§7. The components of the amplitude equation. To complete the amplitude equation in (3.10) it is necessary to determine the fundamental $V_{3}^{(1,1)} e^{i \beta z} E$ contribution to $\partial V_{3} / \partial Y$. As explained previously we shall retain only the nonlinear forcing at this stage, leaving the linear contribution to the amplitude equation to a brief discussion in $\$ 8$.

The equation for $\partial^{2} V_{3} / \partial Y^{2}$ is

$$
\begin{aligned}
\left(c_{0} \frac{\partial}{\partial x}+b_{1} Y\right. & \left.\frac{\partial}{\partial X}+\frac{\partial}{\partial z}-\frac{\lambda \partial^{2}}{\partial Y^{2}}\right) \frac{\partial^{2} V_{3}}{\partial Y^{2}} \\
= & \frac{\partial}{\partial Y}\left\{\frac{\partial V_{1}}{\partial X} \frac{\partial U_{2}}{\partial Y}+\frac{\partial V_{1}}{\partial z} \frac{\partial W_{2}}{\partial Y}+\frac{\partial V_{2}}{\partial X} \frac{\partial U_{1}}{\partial Y}+\frac{\partial V_{2}}{\partial z} \frac{\partial W_{1}}{\partial Y}\right. \\
+ & 2\left(\frac{\partial U_{1}}{\partial X} \frac{\partial U_{2}}{\partial X}+\frac{\partial U_{1}}{\partial z} \frac{\partial W_{2}}{\partial X}+\frac{\partial W_{1}}{\partial X} \frac{\partial U_{2}}{\partial z}+\frac{\partial W_{1}}{\partial z} \frac{\partial W_{2}}{\partial z}\right) \\
& \left.-U_{1} \frac{\partial^{2} V_{2}}{\partial X \partial Y}-V_{1} \frac{\partial^{2} V_{2}}{\partial Y^{2}}-W_{1} \frac{\partial^{2} V_{2}}{\partial z}\right\}
\end{aligned}
$$

where, on the right-hand side, the linear terms have not been included and any terms that clearly have no $e^{i \beta z} E$ component have been ignored.

Following WLC we write the equation for $\partial^{2} V_{3}^{(1,1)} / \partial Y^{2}$ as

$$
\left(c_{0} \frac{\partial}{\partial x}+i \alpha b_{1} Y+i \beta g_{0}-\frac{\lambda \partial^{2}}{\partial Y^{2}}\right) \frac{\partial^{2} V_{3}^{(1,1)}}{\partial Y^{2}}=\frac{\partial M}{\partial Y}
$$

where $M$ is the sum $M_{0}+M_{1}+M_{2}+M_{3}+M_{4}$ and

$$
\begin{aligned}
M_{0}= & i \frac{\partial}{\partial Y}\left\{A_{+}\left(\alpha U_{2}^{(0,0)}+\beta W_{2}^{(0,0)}\right)+A_{-}\left(\alpha U_{2}^{(0,2)}+\beta W_{2}^{(0,2)}\right)\right. \\
& \left.\quad-\tilde{A}_{+}\left(\alpha U_{2}^{(2,2)}+\beta W_{2}^{(2,2)}\right)+\tilde{A}_{-}\left(\alpha U_{2}^{(2,0)}+\beta W_{2}^{(2,0)}\right)\right\} \\
& +2 i \alpha \frac{\partial U_{1}^{(1,-1)}}{\partial Y} V_{2}^{(0,2)}-4 \alpha^{2} U_{1}^{(1,-1)} U_{2}^{(0,2)}, \\
M_{1}= & i \frac{\partial}{\partial Y}\left\{A_{+}\left(\alpha \tilde{U}_{2}^{(0,0)}+\beta \tilde{W}_{2}^{(0,0)}\right)+A_{-}\left(\alpha \tilde{U}_{2}^{(0,-2)}+\beta \tilde{W}_{2}^{(0,-2)}\right)\right\} \\
M_{2}= & 2 i \alpha \frac{\partial U_{1}^{(1,-1)}}{\partial Y} \tilde{V}_{2}^{(0,-2)}-4 \alpha^{2} U_{1}^{(1,-1)} \tilde{U}_{2}^{(0,-2)}, \\
M_{3}= & 2 i \alpha \frac{\partial \tilde{U}_{1}^{(1,-1)}}{\partial Y} V_{2}^{(2,0)}, \\
M_{4}= & -4 \alpha \beta \tilde{U}_{1}^{(1,-1)} W_{2}^{(2,0)} .
\end{aligned}
$$


In (7.3) the terms $W_{2}^{(0,0)}, W_{2}^{(2,0)}$ are absent in the zero cross-flow case in the symmetric situation with $A_{+}=A_{-}$since then they are identically zero.

The contributions to

$$
\left[\frac{\partial \boldsymbol{V}_{3}^{(1,1)}}{\partial Y}\right]_{-\infty}^{\infty}
$$

from $M_{0}$ to $M_{4}$ are calculated in turn. For each we solve, with $j=0$ to 4 ,

$$
\left(c_{0} \frac{\partial}{\partial x}+i \alpha b_{1} Y+i \beta g_{0}-\frac{\lambda \partial^{2}}{\partial Y^{2}}\right) \frac{\partial^{2} V_{3 j}^{(1,1)}}{\partial Y^{2}}=\frac{\partial M_{j}}{\partial Y}
$$

As in WLC the contribution to (7.4) from $M_{0}$ is zero. That from $M_{1}$ is found to be

$$
\begin{aligned}
{\left[\frac{\partial V_{3,1}^{(1,1)}}{\partial Y}\right]_{-\infty}^{\infty}=} & \frac{4 \pi \beta^{2} \alpha^{3} b_{1}^{3}}{\bar{\alpha}^{2} c_{0}^{5}} \iint_{\Delta_{1}} \xi^{3} K^{(0)}(\xi, \eta) e^{-2 i \mu \xi} \\
& \times A_{+}(x-\xi) A_{-}(x-\xi-\eta) \tilde{A}_{-}(x-2 \xi-\eta) d \xi d \eta \\
& +\frac{4 \pi \beta^{2} \alpha^{3} b_{1}^{3}}{\bar{\alpha}^{2} c_{0}^{5}} \iint_{\Delta_{1}} K^{(0)}(\xi, \eta) \\
& \times\left[\xi^{3}+\xi^{2} \eta+\frac{2 \beta^{2}}{\bar{\alpha}^{2}} \int_{0}^{\eta}\left(\xi^{2}+2 \xi(\eta-\zeta) e^{-2 x(\zeta+3 \xi) \zeta^{2}} d \zeta\right]\right. \\
& \times e^{-2 i \mu(\xi+\eta)} A_{-}(x-\xi) \\
& \times A_{+}(x-\xi-\eta) \tilde{A}_{-}(x-2 \xi-\eta) d \xi d \eta .
\end{aligned}
$$

In (7.6) the region of integration $\Delta_{1}$ is, in the situation where the amplitudes are assumed to decay as $x \rightarrow-\infty$, the whole positive quadrant of the $\xi \eta$-plane. However in the situation where the disturbance is taken to be initiated at $x=$ 0 , which is of more importance to us here, $\Delta_{1}$ is the triangle in the positive quadrant over which the arguments of the amplitude functions in (7.6) are positive, that is, the triangle $2 \xi+\eta<x$. Also $K^{(0)}(\xi, \eta)$ is as defined in (3.68) of WLC, namely

$$
K^{(0)}(\xi, \eta)=e^{-s\left(2 \xi^{3}+3 \xi^{2} \eta\right)}
$$

and (7.6) may be compared with their (3.66) in the limit $\mu=0, A_{+}=A_{-}$.

The quantities $\tilde{U}_{2}^{(0,-2)}, \tilde{V}_{2}^{(0,-2)}$ appearing in $M_{2}$ do not follow easily from (6.5), (6.20); we follow WLC by making use of equation (4.6b) relating them and write

$$
\frac{\partial^{2} V_{3,2}^{(1,1)}}{\hat{o} Y^{2}}=Q_{2}(Y, x)-\frac{2 i \alpha}{b_{1}} \tilde{U}^{(0,-2)} \frac{\partial^{2} U_{1}^{(1,-1)}}{\partial Y^{2}}
$$


It is found that $Q_{2}$ satisfies

$$
\begin{aligned}
& \left(c_{0} \frac{\partial}{\partial x}+i \alpha b_{1} Y+i \beta g_{0}-\frac{\lambda \partial^{2}}{\partial Y^{2}}\right) Q_{2} \\
& =2 i \alpha\left\{\frac{\partial U_{1}^{(1,-1)}}{\partial Y} \frac{\partial \tilde{V}_{2}^{(0,-2)}}{\partial Y}+2 i \alpha U_{1}^{(1,-1)} \frac{\partial \tilde{U}_{2}^{(0,-2)}}{\partial Y}\right. \\
& \left.\quad+\frac{i \beta^{2} \alpha b_{1}}{c_{0}^{2} \bar{\alpha}^{2}} A_{+} \tilde{W}_{0}^{(1,-1)} \frac{\partial^{2} U_{1}^{(1,-1)}}{\partial Y^{2}}-\frac{2 \lambda}{b_{1}} \frac{\partial^{3} U_{1}^{(1,-1)}}{\partial Y^{3}} \frac{\partial \widetilde{U}_{2}^{(0,-2)}}{\partial Y}\right\} .
\end{aligned}
$$

As (7.6) contains the amplitude $\tilde{A}_{-}(x-2 \xi-\eta)$ multiplying $A_{ \pm}(x-\xi) A_{\mp}(x-\xi-\eta)$ with unequal kernels, so does the result for $\int_{-\infty}^{\infty} Q_{2} d Y$. We solve for $Q_{2}$ in (7.9) and find finally that

$$
\begin{aligned}
\int_{-\infty}^{\infty} Q_{2} d Y=\frac{8 \pi \beta^{4} \alpha^{3} b_{1}^{3}}{\bar{\alpha}^{4} c_{0}^{5}}\left\{\iint_{\Delta_{1}}\right. & \\
& K_{1}^{(2)}(\xi, \eta) A_{+}(x-\xi) \\
& \times A_{-}(x-\xi-\eta) \tilde{A}_{-}(x-2 \xi-\eta) e^{-2 i \mu \xi} \\
& +K_{2}^{(2)}(\xi, \eta) A_{-}(x-\xi) A_{+}(x-\xi-\eta) \\
& \left.\left.\times \tilde{A}_{-}(x-2 \xi-\eta) e^{-2 i \mu(\xi+\eta)}\right] d \xi d \eta\right\},
\end{aligned}
$$

where

$$
\begin{aligned}
K_{1}^{(2)}(\xi, \eta)= & K^{(1)}(\xi, \eta) \int_{0}^{\xi}[(\eta+\xi)(\eta+3 \zeta) \\
& -(\xi+\eta)(\xi+\eta+2 \zeta)] e^{-3 s(\xi+\eta)(2 \eta+\zeta) \zeta} d \zeta \\
& +\frac{4 \beta^{2}}{\bar{\alpha}^{2}} K^{(1)}(\xi, \eta) \int_{0}^{\xi} e^{-3 s(\xi+\eta)(2 \eta+\zeta) \zeta} \\
& \times \int_{0}^{\zeta}(v-\zeta)\left[1+6 s(\xi-\zeta)(\eta+\zeta)^{2}\right] e^{-s(2 v+3 \xi+3 \eta) v^{2}} d v d \zeta, \\
K_{2}^{(2)}(\xi, \eta)= & K^{(0)}(\xi, \eta) \int_{0}^{\xi}[\zeta(2 \eta+3 \zeta)-\xi(\xi+2 \eta+2 \zeta)] e^{-3 s \xi \zeta^{2}} d \zeta \\
& +\frac{4 \beta^{2}}{\bar{\alpha}^{2}} K^{(0)}(\xi, \eta) \int_{0}^{\xi} e^{-3 s \xi \zeta^{2}} \\
& \times \int_{0}+\zeta \\
& \\
& \\
& \\
& \\
&
\end{aligned}
$$


and

$$
K^{(1)}(\xi, \eta)=e^{-s\left[\xi^{3}+\eta^{3}+(\xi+\eta)^{3}\right]},
$$

and $K^{(0)}$ is defined in (7.7). Comparison of (7.10), (7.11), (7.12) with (3.75) of WLC shows how their contribution to the kernel has split between the unequal $A_{+}, A_{-}$.

To solve (7.5) with $j=3$ write

$$
\frac{\partial^{2} V_{3,3}^{(1,1)}}{\partial Y^{2}}=Q_{3}(Y, x)+\frac{1}{b_{1}} \frac{\partial^{2} \tilde{U}_{1}^{(1,-1)}}{\partial Y^{2}} \frac{\partial V_{2}^{(2,0)}}{\partial Y}
$$

to find that $Q_{3}$ satisfies

$$
\begin{gathered}
\left(c_{0} \frac{\partial}{\partial x}+i \alpha b_{1} Y+i \beta g_{0}-\frac{\lambda \partial^{2}}{\partial Y^{2}}\right) Q_{3}=\frac{2 \beta^{2} \alpha^{2} b_{1}}{\bar{\alpha}^{2} c_{0}^{2}} \frac{\partial^{2} \tilde{U}_{1}^{(1,-1)}}{\partial Y^{2}}\left[A_{+} \chi_{1}^{-}+A-\chi_{1}^{+}+\frac{4 \beta^{2}}{\bar{\alpha}^{2}} \chi_{0}^{+} \chi_{0}^{-}\right] \\
+\frac{2 \lambda}{b_{1}} \frac{\partial^{3} \tilde{U}^{(1,-1)}}{\partial Y^{3}} \frac{\partial^{2} V_{2}^{(2,0)}}{\partial Y^{2}} .
\end{gathered}
$$

After solving equation (7.15) we find, analogously to (7.10), that

$$
\begin{aligned}
& \int_{-\infty}^{\infty} Q_{3} d Y=\frac{8 \pi \beta^{4} \alpha^{3} b_{1}^{3}}{\bar{\alpha}^{4} c_{0}^{5}}\left\{\iint_{\Delta_{1}} K^{(3)}(\xi, \eta)\right. \\
& \\
& \times\left[A_{+}(x-\xi) A_{-}(x-\xi-\eta)\right. \\
& \times \tilde{A}_{-}(x-2 \xi-\eta) e^{-2 i \mu \xi} \\
&+A_{-}(x-\xi) A_{+}(x-\xi-\eta) \\
&\left.\left.\times \tilde{A}_{-}(x-2 \xi-\eta) e^{-2 i \mu(\xi+\eta)}\right] d \xi d \eta\right\}
\end{aligned}
$$

where

$$
\begin{aligned}
K^{(3)}(\xi, \eta)= & K^{(1)}(\xi, \eta) \int_{0}^{\xi} \eta \zeta\left[1+6 s(\xi+\eta+\zeta)^{2}(\xi-\zeta)\right] \Pi_{0}(\xi, \eta, \zeta) d \zeta \\
& +\frac{4 \beta^{2}}{\bar{\alpha}^{2}} \int_{0}^{\xi} \Pi_{0}(\xi, \eta, \zeta) \int_{0}^{\zeta}(\zeta-v) \\
& \times\left[1+6 s(\xi+\eta+\zeta)^{2}(\xi-\zeta)\right] e^{s\left(2 v^{3}+3 \eta v^{2}\right)} d v d \zeta
\end{aligned}
$$

and

$$
\Pi_{0}(\xi, \eta, \zeta)=e^{-s\left(4 \xi^{3}+6 \xi \zeta^{2}+9 \eta \zeta^{2}+6 \xi \eta \zeta+6 \eta^{2} \zeta\right)}
$$

We note that (7.17) is exactly the kernel contribution of WLC (3.81) and, in contrast to the result of (7.10), it has split equally between the unequal $A_{+}, A_{-}$. 
The final requirement is the solution of (7.5) with $j=4$. On substitution for $W_{2}^{(2.0)}$ in (7.3e) from (6.16) we obtain

$$
\begin{aligned}
\int_{-\infty}^{\infty} \frac{\partial^{2} V_{3,4}^{(1,1)}}{\partial Y^{2}} d Y= & \frac{8 \pi \beta^{4} \alpha^{3} b_{1}^{3}}{\bar{\alpha}^{4} c_{0}^{5}} \iint_{\Delta_{1}} K^{(4)}(\xi, \eta) e^{-2 i \mu \xi} \\
& \times\left[A_{+}(x-\xi) A_{-}(x-\xi-\eta)-A_{-}(x-\xi) A_{+}(x-\xi-\eta) e^{-2 i \mu \eta}\right] \\
& \times \tilde{A}_{-}(x-2 \xi-\eta) d \xi d \eta
\end{aligned}
$$

where

$$
K^{(4)}(\xi, \eta)=K_{1}(\xi, \eta) \int_{0}^{\xi} \eta(\xi-\zeta) \Pi_{0}(\xi, \eta, \zeta) d \zeta
$$

As expected the contribution from $M_{4}$ given in (7.19) vanishes when $\mu=0$ and $A_{+}=A_{-}$.

Addition of (7.6), (7.10), (7.16) and (7.19) yields

$$
\begin{aligned}
{\left[\frac{\partial V_{3}^{(1,1)}}{\partial Y}\right]_{-\infty}^{\infty}=\frac{4 \pi \beta^{2} \alpha^{3} b_{1}^{3}}{\bar{\alpha}^{2} c_{0}^{5}}\left\{\iint_{\Delta_{1}}\right.} & e^{-i \mu(2 \xi+\eta)} \\
\times & {\left[K_{1}(\xi, \eta) A_{+}(x-\xi) A_{-}(x-\xi-\eta) e^{i \mu \eta}\right.} \\
& \left.+K_{2}(\xi, \eta) A_{-}(x-\xi) A_{+}(x-\xi-\eta) e^{-i \mu \eta}\right] \\
& \left.\times \tilde{A}_{-}(x-2 \xi-\eta) d \xi d \eta\right\}
\end{aligned}
$$

where

$$
\begin{aligned}
& K_{1}(\xi, \eta)=\xi^{3} K_{0}(\xi, \eta) \\
& +\frac{2 \beta^{2}}{\bar{\alpha}^{2}}\left\{K^{(1)}(\xi, \eta) \int_{0}^{\xi} \eta \zeta\left[1+6 s(\xi-\zeta)(\xi+\eta+\zeta)^{2}\right] \Pi_{0}(\xi, \eta, \zeta) d \zeta\right. \\
& +K^{(1)}(\xi, \eta) \int_{0}^{\xi}[(\eta+\zeta)(\eta+3 \zeta) \\
& \quad-(\xi+\eta)(\xi+\eta+2 \zeta)] e^{-3 s(\xi+\eta)(2 \eta+\zeta) \zeta} d \zeta \\
& \left.+K^{(1)}(\xi, \eta) \int_{0}^{\xi} \eta(\xi-\zeta) \Pi_{0}(\xi, \eta, \zeta) d \zeta\right\}
\end{aligned}
$$




$$
\begin{aligned}
& +\frac{8 \beta^{4}}{\bar{\alpha}^{4}}\left\{K^{(1)}(\xi, \eta) \int_{0}^{\xi} \Pi_{0}(\xi, \eta, \zeta) \int_{0}^{\zeta}(\zeta-v)\right. \\
& \times\left[1+6 s(\xi l-\zeta)(\xi+\eta+\zeta)^{2}\right] e^{s(2 v+3 \eta) v^{2}} d v d \zeta \\
& +K^{(1)}(\xi, \eta) \int_{0}^{\zeta} e^{-3 s(\zeta+\eta)(2 \eta+\zeta) \zeta} \int_{0}^{\zeta}(v-\zeta) \\
& \left.\times\left[1+6 s(\xi-\zeta)(\eta+\zeta)^{2}\right] e^{-s(2 v+3 \xi+3 \eta) v^{2}} d v d \zeta\right\}
\end{aligned}
$$

and

$$
\begin{aligned}
& K_{2}(\xi, \eta)=K_{0}(\xi)\left(\xi^{3}+\xi^{2} \eta\right) \\
& +\frac{2 \beta^{2}}{\bar{\alpha}^{2}}\left\{K_{0}(\xi, \eta) \int_{0}^{\eta}\left[\xi^{2}+2 \xi(\eta-\zeta)\right] e^{-s(2 \zeta+3 \xi) \zeta^{2}} d \zeta\right. \\
& +K_{0}(\xi, \eta) \int_{0}^{\xi}[\zeta(2 \eta+3 \zeta)-\xi(\xi+2 \eta+2 \zeta)] e^{-3 . \xi \xi \zeta^{2}} d \zeta \\
& +K_{1}(\xi, \eta) \int_{0}^{\xi} \eta \zeta\left[1+6 s(\xi-\zeta)(\xi+\eta+\zeta)^{2}\right] \Pi_{0}(\xi, \eta, \zeta) d \zeta \\
& \left.-K_{1}(\xi, \eta) \int_{0}^{\xi} \eta(\xi-\zeta) \Pi_{0}(\xi, \eta, \zeta) d \zeta\right\} \\
& +\frac{8 \beta^{4}}{\bar{\alpha}^{4}}\left\{K_{0}(\xi, \eta) \int_{0}^{\xi} e^{-3 s \xi \zeta^{2}} \int_{0}^{\eta+\zeta}(v-\eta-\zeta)\right. \\
& \times\left[1+6 s(\xi-\zeta) \zeta^{2}\right] e^{-s(2 v+3 \xi) v^{2}} d v d \zeta \\
& +K_{1}(\xi, \eta) \int_{0}^{\xi} \Pi_{0}(\xi, \eta, \zeta) \int_{0}^{\zeta}(\zeta-v) \\
& \left.\times\left[1+6 s(\xi-\zeta)(\xi+\eta+\zeta)^{2}\right] e^{s(2 v+3 \eta) v^{2}} d v d \zeta\right\}
\end{aligned}
$$

Equations (7.22), (7.23) illustrate the split of the kernel (3.85) of WLC when $A_{+} \neq A_{-}$. It is not clear that the form of the split could have been anticipated without undertaking the details of the calculation. The final term in the braces 
with factor $2 \beta^{2} / \bar{\alpha}^{2}$ does not appear in $K_{1}+K_{2}$ so is not evident in the kernel when $A_{+}=A_{-}$and $\mu=0$.

In the next section we assemble the contributions to the amplitude equations and discuss their possible forms, boundary conditions and termination before finally presenting numerical solutions in $\$$.

\$8. The amplitude equations. The final amplitude equations are obtained by matching $\left[\partial V_{3}^{(1,1)} / \partial Y\right]_{-\infty}^{\infty}$ from the critical-layer solution to the jump in $\partial v_{2}^{(1,1)} / \partial y$ predicted by the core in (3.8). This is the difference $J_{+}^{(1,1)}-J_{-}^{(1,1)}$ as set out in (3.10). In the discussion of the critical layer we did not specifically display the linear forcing terms because, as is customary in studies involving linear critical layers, whether viscous or non-equilibrium or both, the effect of such terms is that the logarithm in (3.8) increases by $i \pi \operatorname{sgn} b_{1}$ as $y$ increases through $y_{c}$ and there is an addition to the jump as specified in (4.11). We define

$$
B_{ \pm}(x)=e^{\mp i \mu x} A_{ \pm}(x)
$$

and write the resulting equations as

$$
\begin{aligned}
C \frac{d B_{ \pm}}{d x} \pm F B_{ \pm}(x)+ & \Gamma \iint_{\Delta_{1}} \widetilde{B}_{\mp}(x-2 \xi-\eta) \\
\times & \left\{K_{1}(\xi, \eta) B_{ \pm}(x-\xi) B_{\mp}(x-\xi-\eta)\right. \\
& \left.+K_{2}(\xi, \eta) B_{\mp}(x-\xi) B_{ \pm}(x-\xi-\eta)\right\} d \xi d \eta=0
\end{aligned}
$$

where, in (8.2), the kernels $K_{1}, K_{2}$ are given in (7.22), (7.23), $\Gamma=4 \pi \beta^{2} \alpha^{3} b_{1}^{3} /$ $\left(\bar{\alpha}^{2} c_{0}^{5}\right)$ and $C, F$ are constants obtained from the outer solution. If we denote the two integrals appearing in (3.10) by $J_{1}, J_{2}$ where

$$
\begin{aligned}
& J_{1}=\int_{0}^{\infty}\left(\frac{c_{0} U_{0}^{\prime \prime}}{\left(U_{0}-c_{0}\right)^{2}}-2 \alpha^{2}\right)\left(v_{1}^{(1)}\right)^{2} d y, \\
& J_{2}=\int_{0}^{\infty}\left(W_{0}^{\prime \prime}-\frac{W_{0} U_{0}^{\prime \prime}}{U_{0}-c_{0}}\right) \frac{\left(v_{1}^{(1)}\right)^{2}}{U_{0}-c_{0}} d y,
\end{aligned}
$$

then

$$
\begin{aligned}
& C=\frac{i J_{1}}{\alpha}-\frac{c_{0} b_{3} \pi}{\alpha b_{1}^{2}}, \\
& F=\frac{\beta}{\alpha}\left(J_{2}+\frac{g_{0} J_{1}}{c_{0}}+\frac{i g_{2} \pi}{b_{1}}\right) .
\end{aligned}
$$

The constants $g_{0}, g_{2}, b_{3}$ were introduced in (4.3), and the presence of the terms in $\pi$ confirms that the linear contribution to (8.2) has been incorporated. We note that the constant $F$ embodies the cross-flow contribution and precludes the existence of a solution of (8.2) with equal amplitude waves $B_{+}=B_{-}$. 
The expressions described in the previous sections can be generalized to include the case where the wave is generated at a small, $O\left(\varepsilon^{2}\right)$, distance downstream of the neutral position taken here as $x^{*}=0$. In this instance the expansions are made about the point $x^{*}=\varepsilon^{2} x_{0}$ and the analysis described previously follows through, the minor changes affecting the linear terms only. With this modification equation (8.2) becomes

$$
C \frac{d B_{ \pm}}{d x}+(\tau L \pm F) B_{ \pm}-\Gamma N\left(B_{ \pm}, B_{\mp}\right)=0
$$

where $N$ denotes the non-linear term, $\tau$ is proportional to $x_{0}$, the distance from criticality, and $L$ is a complex constant derived from an integral of the basic profile $U_{0}(y)$. This extra term, which is the same for both equations in (8.5), was introduced by Goldstein and Choi (1989) (see also WLC) to allow the nonlinear solutions to (8.5) to match back, as $x \rightarrow-\infty$, with an initial region of exponentially growing waves.

Comments concerning the behaviour of $B_{ \pm}$as $x \rightarrow-\infty$ are applicable to the situation when $\Delta_{1}$ in (8.2) is the whole positive quadrant in the $\xi \eta$-plane. The preceding analysis is appropriate to that case but we see from (8.5) that a sufficiently large $\tau$ with

$$
\operatorname{Re}((\tau L \pm F) / C)<0
$$

must be chosen if both solutions of (8.5) are to decay as $x \rightarrow-\infty$. In the absence of cross-flow the wave-number pairs $(\alpha, \pm \beta)$ both lie on the neutral stability curve and any non-zero $\tau$ enables the analysis to be undertaken in the unstable region. However, when cross-flow is present, the neutral curve is perturbed so that one of the pairs moves into the unstable region and the other into the stable region. The condition (8.6) may be interpreted as requiring that the distance into the unstable region is large enough to counteract the perturbations to the neutral curve and thus to force the wave-number pairs to remain within the unstable region.

The possible behaviour of the solutions of (8.2) or (8.5) as $x \rightarrow-\infty$ is not, however of great concern to us. We are anticipating a scenario in which $\Delta_{1}$ is the triangle $2 \xi+\eta<x$ in the positive quadrant of the $\xi \eta$-plane and $B_{ \pm}(0)$ are prescribed. As noted after (5.10), and pointed out by Hickernell (1984), further terms are thus introduced into the core flow. Eigensolutions of the core flow equations may be constructed with arbitrary functions whose determination is expected to require a specification of the exact setting up of the waves considered here. The additional solutions may be regarded as transients of such an initial value problem and do not affect the main result, equation (8.2), of this paper.

$\$ 9$ Numerical results. In this section results of numerical integrations of equations (8.5) for representative choices of the coefficients are described. To reduce the number of possibilities, $B_{ \pm}$and $x$ are rescaled so that $\Gamma= \pm 1$ and $\operatorname{Re} F=1$. The kernels $K_{1}(\xi, \eta), K_{2}(\xi, \eta)$ were calculated numerically using Simpson's rule for the viscous case $s=1$ with a grid spacing of 0.01 . In the 
inviscid limit with $s=0$ they reduce to

$$
\begin{aligned}
& K_{1}(\xi, \eta)=\left(1-\frac{2 \beta^{2}}{\bar{\alpha}^{2}}\right) \eta^{3}, \\
& K_{2}(\xi, \eta)=\xi^{3}+\xi^{2} \eta+\frac{2 \beta^{2}}{\bar{\alpha}^{2}}\left(\eta^{2}-\xi^{2}\right) \xi-\frac{4 \beta^{4}}{\bar{\alpha}^{4}} \xi \eta(\xi+\eta) .
\end{aligned}
$$

Solutions of the amplitude equations are presented for the chosen set of coefficients:

$$
C=1-\frac{1}{4} i, \quad F=1+\frac{1}{2} i, \quad \frac{\beta^{2}}{\bar{\alpha}^{2}}=\frac{1}{4} .
$$

Calculations were performed for other choices of these coefficients but showed only quantitative rather than qualitative changes in the solution behaviour.

Figures $1 \mathrm{a}, \mathrm{b}$ show inviscid solutions for $\Gamma= \pm 1$ respectively for $\tau=0$. The initial conditions were $B_{+}(0)=B_{-}(0)=1$ but the non-zero cross-flow parameter $F$ in (9.2) forces the amplitudes to develop unequally. As can be seen, in both cases there is an initial linear stage involving exponential decay of $B_{+}$and growth of $B_{-}$followed by nonlinear interaction which forces rapid growth of $B_{+}$. Indeed if, in the linear growth/decay stage, $B_{\mp} \propto e^{ \pm \delta x}$, the non-linear interaction raises $B_{+}$from an exponential decay to a magnitude $O\left(x^{4} e^{\delta x}\right)$. It can be seen in all computations presented that $\left|B_{+}\right|$overtakes $\left|B_{-}\right|$soon after the mutual interaction occurs. This explosive growth of both amplitudes is rapidly followed by termination in an algebraic singularity that is essentially identical to that of Goldstein and Choi (1989), and takes the form $B_{ \pm} \approx a_{0}\left(x_{s}-x\right)^{-(3+i \sigma)}$ as $x \rightarrow x_{s}-0$ where $\sigma$ is a real and $a_{0}$ a complex constant satisfying

$$
\begin{aligned}
C(3+i \sigma)= & \Gamma\left|a_{0}\right|^{2} \int_{0}^{\infty} \int_{0}^{\infty} K(\xi, \eta) \\
& \times[(1+\xi)(1+\xi+\eta)]^{-(3+i \sigma)}(1+2 \xi+\eta)^{-(3-i \sigma)} d \xi d \eta
\end{aligned}
$$

In (9.3), $K=K_{1}+K_{2}$ where $K_{1}, K_{2}$ take their inviscid form as in (9.1). At this point it is worth noting that for fixed values of the other parameters in (8.5), the change of sign of $\Gamma$ does not affect the final outcome, but does influence the behaviour immediately prior to the singularity in that the amplitudes become highly oscillatory when $\Gamma$ is positive.

In Figs $2 \mathrm{a}, \mathrm{b}$ results are presented for the viscous case $s=1$ and $\Gamma= \pm 1$ respectively. Again $B_{+}(0)=B_{-}(0)=1$ and we observe that, as noted by WLC, the main effect of the viscosity is to delay, but not prevent, the formation of the singularity. Figures $3 a, b$ illustrate the effect of changing the initial conditions to $B_{+}(0)=2, B_{-}(0)=1$. 

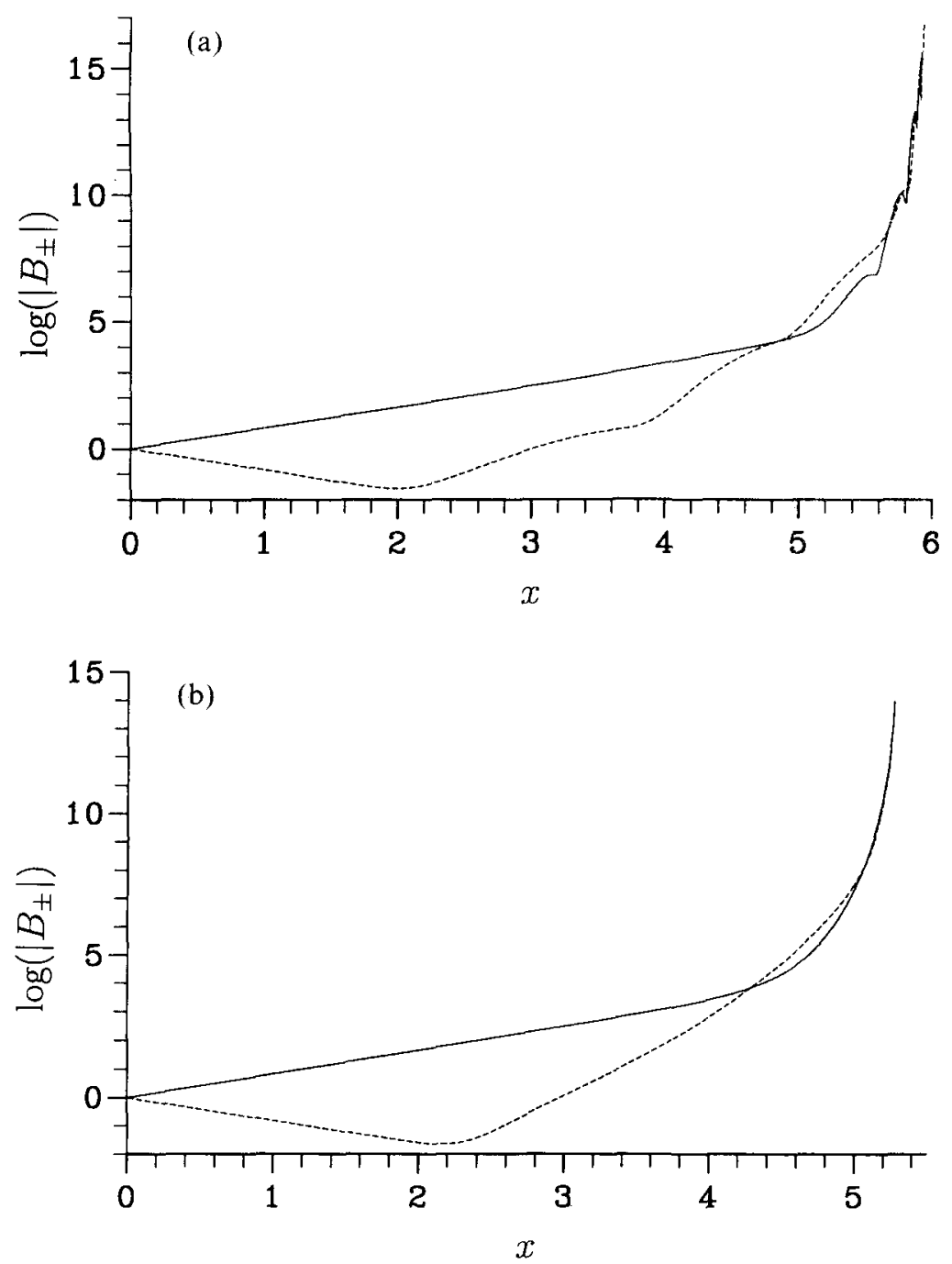

Figure 1. Solutions of (8.5) in the inviscid limit $s=0$ with (a) $\Gamma=1$ and (b) $\Gamma=-1$. The solid and dashed lines denote the solutions $B_{-}$and $B_{+}$respectively.

Finally, in Figs $4 \mathrm{a}, \mathrm{b}$ we show a specimen case with non-zero $\tau$ so that the solution may be matched as $x \rightarrow-\infty$ to an initial linear exponentially growing stage. For illustration, here $\tau=1$ and $L=-2.5$ to ensure that (8.6) is satisfied. Here both $B_{+}$and $B_{-}$decay as $x \rightarrow-\infty$ but, as with solutions initiated at $x=0$ as discussed above, after the waves have grown to a sufficiently large amplitude, similar nonlinear interactions take place and the solutions again terminate in a singularity.

In their computations of (8.5) with $F=0, B_{+}=B_{-}$and decaying solutions as $x \rightarrow-\infty$, WLC found that for sufficiently large values of $\lambda$, the viscous parameter, it was possible to obtain solutions with exponential decay as $x \rightarrow \infty$ 

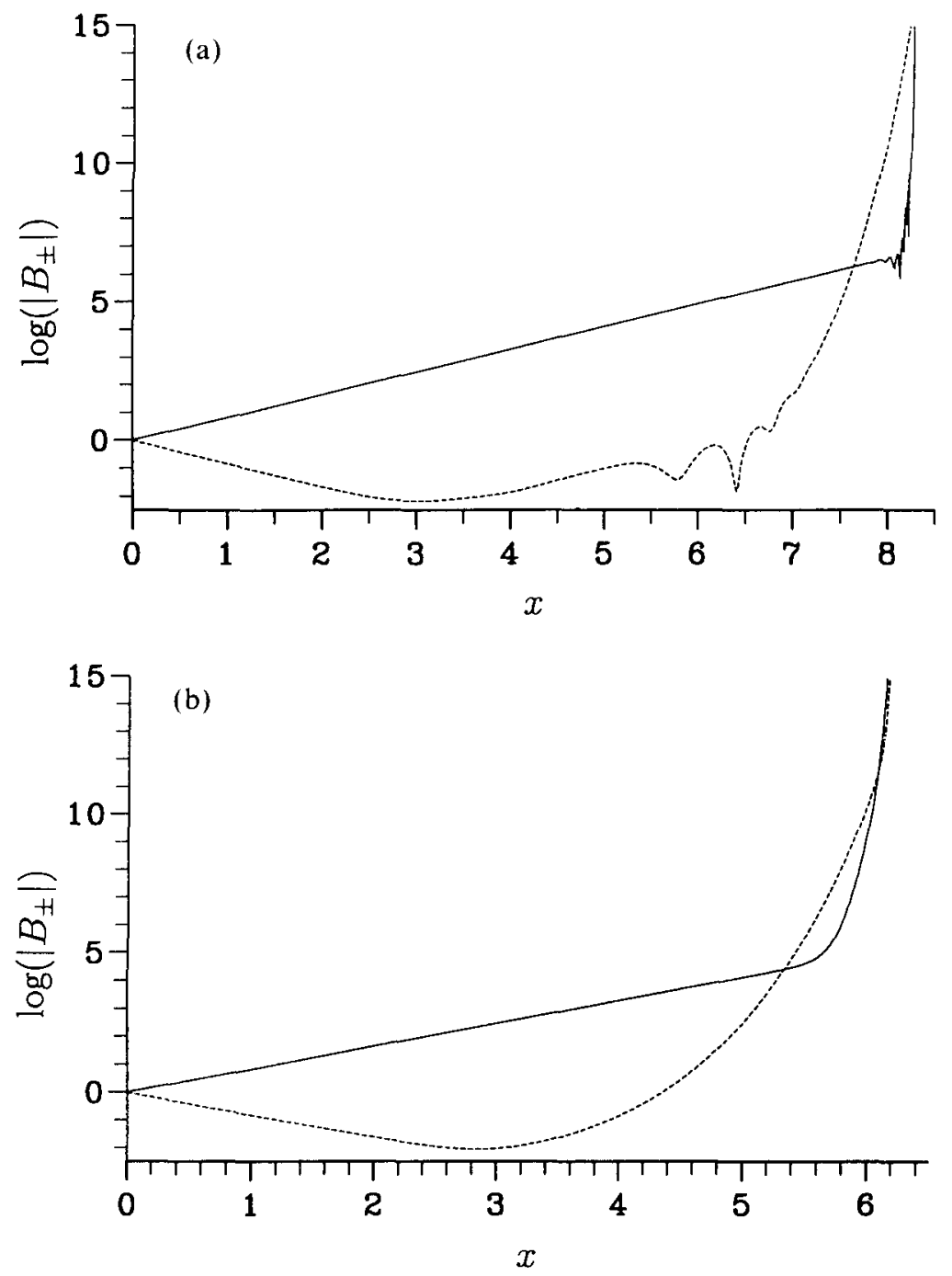

Figure 2. Viscous solutions of (8.5) when $s=1$ with (a) $\Gamma=1$ and (b) $\Gamma=-1$.

rather than a terminal singularity at a finite value of $x$. They showed that this was not unexpected in that, as $\lambda \rightarrow \infty$, they were able to show that their amplitude equation reduced to a form of that of SBB with the non-parallel term there replaced by a constant. This was known to possess decaying solutions for certain values of the coefficients. Indeed, they found a simple solution of their limiting equation demonstrating the property. An analysis similar to theirs may be applied to (8.5), to show that as $\lambda \rightarrow \infty$, it reduces to the pair of equations derived by Brown and Smith (1996) for cross-flow of $O\left(\varepsilon^{3}\right)$, with the non-parallel term there either absent (in the case $\tau=0$ ) or replaced by a constant. No decaying solutions of that system were encountered, and it may be shown that no decaying solutions of the corresponding limiting system obtained here can exist either with $\tau=0$ and initial conditions at $x=0$, or with 

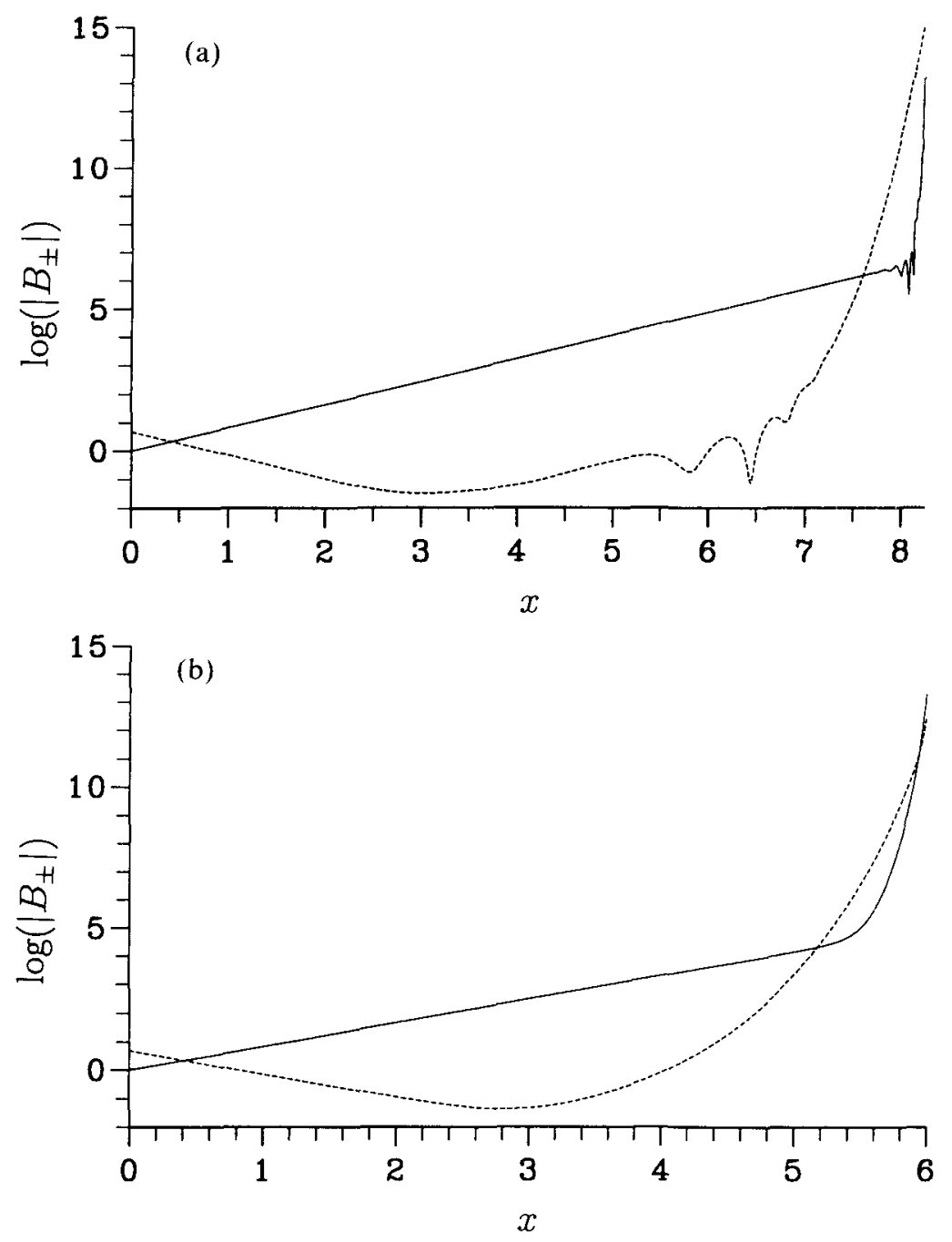

Figure 3. As figure 2 but with different initial conditions.

$\tau \neq 0$ and conditions of exponential decay at minus infinity. Again, for the particular case of $\beta^{2} / \bar{\alpha}^{2}=\frac{1}{2}$, as in WLC, the non-linear terms in (8.5) vanish in both limits $\lambda=0$ and $\lambda=\infty$, and for large but finite $\lambda$, WLC encountered solutions that settled into a periodic form as $x$ (their $t$ ) increased. Whether analogous periodic solutions exist in the present non-symmetric cross-flow situation has not been established.

10. Conclusions. In this fixed-frequency study of the non-linear interaction of two waves of unequal amplitude in a predominantly two-dimensional boundary layer with small cross-flow we have concentrated on disturbances initiated at an inflexion point of the basic flow ( $c f$. the spatio-temporal formulations developed in Savin (1996), Smith (1996) for general initial conditions). The 

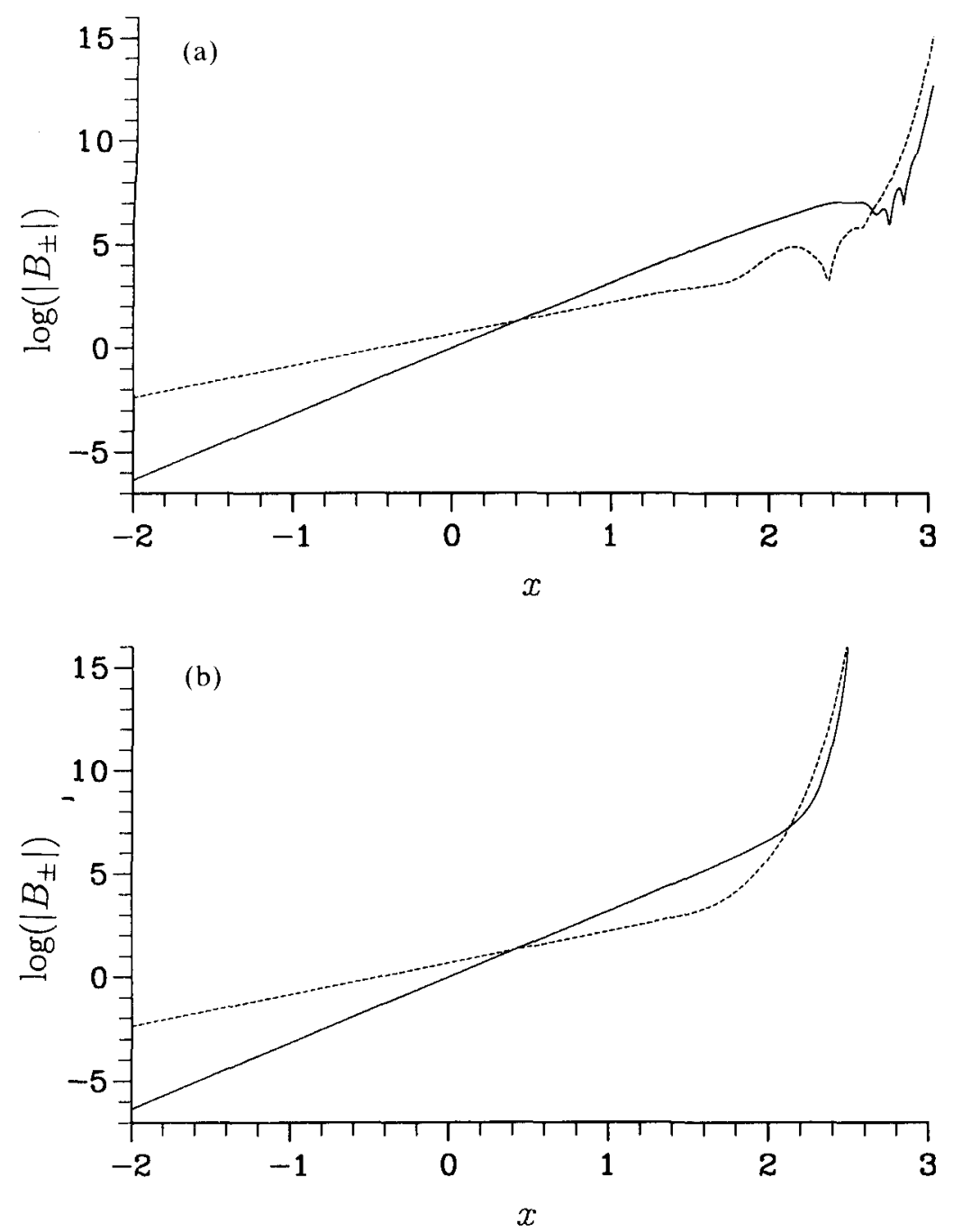

Figure 4. As figure 2 but now solutions are forced to match back to an initial linearly-growing stage by a positive value of the $\tau$ parameter.

critical layer is of non-equilibrium type in $x$ and forces an adjustment to the basic flow in a manner that is now well understood. Although there have been many studies of boundary-layer instability and its interpretation as a precursor of laminar/turbulent transition, some of which are noted here in the Introduction, the present work essentially represents an extension of the work of Brown and Smith (1996) to higher values of the cross-flow. The scalings involved take the investigation into the parameter range of WLC and the analysis has intentionally been set into their mould to permit cross-checking where applicable.

The resulting coupled amplitude equations are interpreted as an initialvalue problem in the manner of Hickernell (1984) and are solved numerically for representative values of the constant coefficients. All solutions terminated 
with the singularity of Goldstein and Choi (1989) although viscosity delayed its onset. Presumably the singularity is smoothed out in the equivalent of an Euler region, the study of which is beyond the scope of this paper. As the viscosity parameter $\lambda \rightarrow \infty$ the limiting situation is that of Brown and Smith (1996), while as the cross-flow parameter $g_{0}$ in equation (5.4) increases it seems that the critical layer divides into two separated layers although no account has been taken here of this observation. It is intended to exploit this in future work. The study differs from those of Gajjar $(1995,1996)$ in that, although the cross-flow here is of smaller magnitude, the speed of the basic flow at the critical layer position is not necessarily small, nor is the angle between the imposed waves.

Acknowledgement. Support for T.A. from the EPSRC Mathematics Committee is gratefully acknowledged.

\section{References}

T. Allen, S. N. Brown and F. T. Smith. On vortex/wave interactions. Part 2: Originating from axisymmetric flow with swirl. J. Fluid Mech., 325 (1996), 145161.

A. P. Bassom and J. S. B. Gajjar. Non-stationary cross-flow vortices in three-dimensional boundary-layer flows. Proc. R. Soc. Lond. A, 417 (1988), 179. 212.

D. J. Benney and C. Chow. A mean flow first harmonic theory for hydrodynamic instabilities. Stud. Appl. Maths., 80 (1989), 37-73.

P. G. Brown, S. N. Brown, F. T. Smith and S. N. Timoshin. On the starting process of strongly nonlinear vortex/Rayleigh-wave interactions. Mathematika, 40 (1993), 729.

S. N. Brown and F. T. Smith. On vortex/wave interactions. Part 1: Non-symmetric input and cross-flow in boundary layers. J. Fluid Mech., 307 (1996), 101133.

D. A. R. Davis and F. T. Smith. Influence of cross-flow on nonlinear Tollmien Schlichting/vortex interaction. Proc. R. Soc. Lond. A, 446 (1994), 319340.

J. S. B. Gajjar. On the nonlinear evolution of a stationary cross-flow vortex in a fully threedimensional boundary layer flow. NASA Contractor Report, 198405 (1995).

J. S. B. Gajjar. Nonlinear stability of non-stationary cross-flow vortices in compressible boundary layers. Stud. Appl. Math., 96 (1996), 53-84.

M. E. Goldstein and S.-W. Choi. Nonlinear evolution of interacting oblique waves on two-dimensional shear layers. J. Fluid Mech., 207 (1989), 97 120.

M. E. Goldstein and S. J. Leib. Nonlinear evolution of oblique waves on compressible shear layers. J. Fluid Mech., 207 (1989), 73-96.

P. Hall and F. T. Smith. The nonlinear interaction of Tollmien-Schlichting waves and TaylorGörtler vortices in curved channel flow. Proc. R. Soc. Lond. A, 417 (1988), 255-282.

P. Hall and F. T. Smith. Nonlinear Tollmien-Schlichting/vortex interaction in boundary layers. Eur. J. Mech. B/Fluids, 8 (1989), 179-205.

P. Hall and F. T. Smith. Proc. ICASE workshop on instability and transition, vol. II, M. Y. Hussaini \& R. G. Voigt, eds., (Springer, 1990), 539.

P. Hall and F. T. Smith. On strongly nonlinear vortex/wave interactions in boundary layer transition. J. Fluid Mech., 227 (1991), 641666.

F. J. Hickernell. Time-dependent critical layers in shear flows on the beta plane. J. Fluid Mech., 142 (1984), 431-449.

D. J. Savin. Linear and nonlinear aspects of interacting boundary-layer transition. Ph.D. Thesis (University of London, 1996).

F. T. Smith. On transition over surface roughnesses. A.I.A.A. paper no. 96-1992, presented at New Orleans meeting, June 17-20, 1996.

F. T. Smith, S. N. Brown and P. G. Brown. Initiation of three-dimensional non-linear transition paths from an inflectional profile. Eur. J. Mech. B/Fluids, 12 (1993), 447.473.

$\mathrm{X}$. Wu. The non-linear evolution of high-frequency resonant-triad waves in an oscillatory Stokes' layer at high Reynolds number. J. Fluid Mech., 245 (1992), 553597.

X. Wu, Private Communication. (1996).

$\mathrm{X}$. Wu, S. S. Lee. and S. J. Cowley. On the weakly nonlinear three-dimensional instability of shear layers to pairs of oblique waves: The Stokes layer as a paradigm. J. Fluid Mech., 253 (1993), 681721. 
Dr. T. Allen,

Room 247, Ocean Applications,

The Met. Office,

London Road,

Bracknell. RG12 2SZ

Professor S. N. Brown,

Department of Mathematics,

University College London,

Gower Street,

London. WC1E 6BT

Professor F. T. Smith,

Department of Mathematics,

University College London,

Gower Street,

London. WC1E 6BT
76D20: FLUID MECHANICS; Incompressible viscous fuids; Higher-order effects in boundary layers.

Received on the 3rd of February, 1997. 